\title{
Kernos
}

Revue internationale et pluridisciplinaire de religion grecque antique

$26 \mid 2013$

Varia

\section{Laurent Bricault, Richard Veymiers (dir.), Bibliotheca Isiaca II}

Jérôme Masselis

\section{(2) OpenEdition \\ Journals}

Édition électronique

URL : http://journals.openedition.org/kernos/2179

DOI : 10.4000/kernos. 2179

ISSN : 2034-7871

\section{Éditeur}

Centre international d'étude de la religion grecque antique

\section{Édition imprimée}

Date de publication : 10 octobre 2013

Pagination : 440-441

ISSN : 0776-3824

\section{Référence électronique}

Jérôme Masselis, «Laurent Bricault, Richard Veymiers (dir.), Bibliotheca Isiaca II », Kernos [En ligne],

26 | 2013, mis en ligne le 10 octobre 2013, consulté le 02 mars 2021. URL : http://

journals.openedition.org/kernos/2179; DOI : https://doi.org/10.4000/kernos.2179

Ce document a été généré automatiquement le 2 mars 2021.

Kernos 


\title{
Laurent Bricault, Richard Veymiers (dir.), Bibliotheca Isiaca II
}

\author{
Jérôme Masselis
}

\section{RÉFÉRENCE}

Laurent Bricault, Richard Veymiers (dir.), Bibliotheca Isiaca II, Bordeaux, Ausonius éditions, 2011. 1 vol. $21 \times 29,5 \mathrm{~cm}, 486$ p. ISBN : 978-2-35613-053-2.

1 Trois ans après la parution du premier volume ${ }^{1}$, la Bibliotheca Isiaca apparaît comme un outil indispensable pour tout chercheur intéressé par les études isiaques. Placée sous la direction de deux spécialistes de ce champ de recherche très dynamique, Laurent Bricault et Richard Veymiers, cette seconde livraison est construite en quatre parties bien définies, ayant chacune un objectif précis.

2 La première partie correspond aux Actes de la troisième journée $\mathrm{du} I V^{e}$ colloque international sur les études isiaques, qui s'est tenu à Liège en novembre 2008 en hommage à l'un des plus éminents spécialistes du domaine, le Professeur Michel Malaise, et regroupe ainsi plusieurs contributions touchant aux cultes isiaques en Grèce, riches en inédits divers. Après une introduction des deux éditeurs, faisant l'état des recherches pour le territoire grec, Perikles Christodoulou signe une étude sur les reliefs votifs du sanctuaire d'Isis à Dion. Examinant des documents déjà connus, l'A. fait quelques nouvelles observations, réinterprétant notamment l'inscription du relief au buste d'Isis, et nous fournit de précieuses photographies et restitutions. Les trois articles suivants portent sur l'une des plus importantes découvertes faites dans le territoire grec ces quinze dernières années, le sanctuaire des dieux égyptiens à Marathon. Ifigeneia Dekoulakou fait, dans la plus grande partie de sa contribution, une présentation actualisée du site, qu'elle identifie très justement avec le Kanôbos mentionné par Philostrate, et publie pour la première fois les inscriptions qui y ont été trouvées. Pelly Fotiadi se concentre sur un type de lampes rituelles en terre cuite trouvées dans le complexe. Labrini siskou présente toutes les statues égyptisantes 
masculines trouvées à chaque entrée du sanctuaire en s'interrogeant sur leur possible identification à Antinoos-Osiris. Charikleia Fantaoutsaki soumet un rapport préliminaire des fouilles du sanctuaire d'Isis de Rhodes (p. 47-63), qui remonte à la haute époque hellénistique, et s'intéresse tout particulièrement à la crypte, ainsi qu'au caractère égyptisant d'une partie de la statuaire S'intéressant au culte d'Isis à Messène, Petros Themelis identifie le sanctuaire à proximité du théâtre et décrit tous les objets qui présentent, selon lui, un caractère isiaque, les plus notables étant les statues d'Isis à la voile et d'Isis assise, allaitant le petit Harpocrate. Richard Veymiers dresse un bilan de la présence isiaque à Argos, la ville d'Io, intimement liée à l'Égypte, qui semble avoir accueilli ces cultes dès la haute époque hellénistique, avec un ou plusieurs sanctuaires dont la localisation demande encore à être précisée par l'archéologie.

3 La deuxième partie, intitulée Nova Isiaca, contient une douzaine d'articles se concentrant pour la plupart sur des documents inédits ou méconnus. Laurent Bricault signe d'abord une étude sur une statuette en bronze d'Hermanubis dédiée à Arès, dont il décrit l'iconographie avant de proposer une interprétation de l'inscription. Il s'intéresse ensuite à des poids de plomb marqués du basileion d'Isis qui semblent provenir de Byblos, où le culte d'Isis et des membres de son cercle était florissant, malgré l'absence d'inscriptions. Rappelant les problèmes soulevés par les représentations statuaires de Sarapis, Laurent Bricault et Jean-Louis Podvin en réunissent trente-sept exemplaires apparus sur le marché des antiquités ces quinze dernières années. Une telle contribution révèle ô combien la réalisation d'un grand répertoire iconographique serait utile à la recherche. Marie-Christine Budischovsky s'attache d'abord à un groupe en bronze où elle reconnaît un Bès-Héraklès dans une prise de lutte plutôt que Bès portant Harpocrate. Elle publie ensuite deux bronzes à l'effigie d'Harpocrate, le montrant l'un debout avec la cornucopia, l'autre assis sur le bélier. Le livre XI des Métamorphoses (XI, 6-13) d'Apulée évoque l'importance de la rose dans les cultes isiaques, ce que vise à démontrer P. Koemoth. Notant que cette plante a supplanté le grand épilobe auprès d'Isis à la suite de son assimilation à Aphrodite, il y voit le symbole, pour les défuntes isiaques, de leur union à Osiris, d'où la présence de l'hydrie. Michel Malaise s'interroge sur l'attitude du futur Auguste envers les cultes isiaques à Rome en 28 av. J.-C. à partir d'un passage controversé de Dion Cassius. Examinant les traces isiaques de l'Urbs à l'époque républicaine, il éprouve l'hypothèse récente d'E.M. Orlin ${ }^{2}$ qui considère que les sanctuaires restaurés alors par Octave étaient dévolus à Isis et aux membres de son cercle. Michel Malaise s'associe ensuite à Richard Veymiers pour présenter une série de figurines en bronze à l'effigie d'une divinité masculine panthée arborant le basileion d'Isis. De telles images, qui reflètent la volonté des Anciens de pourvoir une divinité de multiples compétences, semblent avoir été diversement perçues selon les circonstances. Après avoir rappelé les liens entre les Lagides et la Cilicie, évoqué la rencontre d'Antoine et Cléopâtre, Sophie Picaud et JeanLouis Podvin examinent les isiaca provenant de Tarse et de sa région. Si aucun temple n'est attesté jusqu'à présent dans la cité proprement dite, les divinités isiaques sont bien représentées dans son monnayage. Ennio Sanzi montre la survivance des divinités isiaques à l'heure des premiers Chrétiens en présentant une série de textes coptes - un matériel méconnu, rarement mobilisé. Ces éléments païens surgissent dans les traditions locales, où ces divinités se sont maintenues sous une forme plus traditionnelle qu'hellénisée. Richard Veymiers propose un premier supplément,

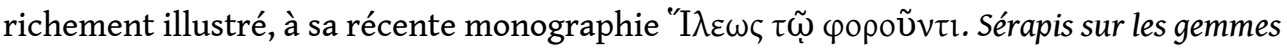


et les bijoux antiques (2009), avec notamment 121 nouvelles pièces, en grande partie inédites.

4 Faisant office de troisième partie, le deuxième Supplément au Recueil des Inscriptions concernant les Cultes Isiaques (RICIS) contient, outre une précieuse mise à jour bibliographique et analytique, 32 textes non recensés jusqu'ici, portant ainsi le corpus épigraphique isiaque à 1845 numéros (p. 273-307). Un index à douze entrées vient judicieusement compléter ce Supplément.

5 Enfin, la précieuse chronique bibliographique répertorie avec un commentaire critique toutes les publications concernant d'une manière ou d'une autre le phénomène isiaque. Cet outil si utile, qui est l'œuvre d'une véritable équipe internationale, propose un complément aux années 2000-2004, parues dans la Bibliotheca Isiaca I, avant de présenter les notices relatives aux années 2005-2008. Ce sont plus de 900 titres qui ont ainsi été chroniqués entre 2000 et 2008, dans le prolongement de l'Inventaire bibliographique des Isiaca (IBIS) de Jean Leclant et Gisèle Clerc. La bibliographie générale est si riche qu'elle peut servir en soi d'instrument de travail pour les chercheurs. Un index général très précis facilite considérablement la consultation.

6 L'importance de ce volume, qui tient tant à la diversité et la qualité de ses contributions qu'aux outils de travail qu'il nous offre, reflète l'extraordinaire dynamisme que connaissent les études isiaques depuis plus de quinze ans ${ }^{3}$, et dont cette collection, la Bibliotheca Isiaca, est devenue la vitrine internationale. S'il atteste la prédominance des chercheurs francophones dans ce domaine de la recherche historique, cet ouvrage, rédigé en français, anglais et italien, s'est assuré aussi le concours des scientifiques issus d'horizons divers. On saluera particulièrement la participation d'archéologues grecs qui ont fait connaitre ici certaines trouvailles tout à fait remarquables. Souhaitons que les prochains volumes de cette belle collection puissent accueillir d'aussi riches contributions.

\section{NOTES}

1. L. Bricault (éd.), Bibliotheca Isiaca I, Bordeaux, 2008. Cf. L. Puccio, in Kernos 22 (2009), p. 336-339.

2. E.M. Orlin, «Octavian and Egyptian Cults: Redrawing the Boundaries of Romanness ", AJPh 129 (2008), p. 231-253.

3. L. Bricault, R. Veymiers, "Quinze ans après. Les études isiaques (1997-2012) : un premier bilan ", in L. Bricault, M.J. Versluys (éd.), Egyptian gods in the Hellenistic and Roman Mediterranean: Image and reality between local and global, Palerme, 2012 (Mythos,suppl. 3), p. 1-23. 


\section{AUTEURS}

JÉRÔME MASSELIS

Université de Toulouse 2 - Le Mirail 